\title{
The motivation produced by morphine and food is isomorphic: Approaches to specific motivational stimuli are learned
}

\author{
KARIM NADER and DEREK VAN DER KOOY \\ University of Toronto, Toronto, Ontario, Canada
}

\begin{abstract}
Using an unbiased place preference paradigm, opiate-naive rats were conditioned as follows: one side was paired with 18-h food deprivation, and the other environment was paired with 18-h food deprivation and morphine ( $20 \mathrm{mg} / \mathrm{kg}$ i.p.). On the test day, animals showed a large preference for the environment paired with hunger and morphine. The acquisition of this preference was unaffected by bilateral ibotenic lesions of the brainstem tegmental pedunculopontine (TPP) nucleus, but was completely blocked by pretreatment with alpha-flupentixol $(0.8 \mathrm{mg} / \mathrm{kg}$ i.p.). The failure of TPP lesions, which have previously been shown to abolish the motivational effects of morphine in drug-naive animals, to affect the present preferences in food-deprived rats suggests that morphine is not producing its normal (drug-naive) motivational effects. We suggest that morphine can substitute for the dopamine-mediated, motivational effects of food in a food-deprived animal.
\end{abstract}

A fundamental question in motivational theory is how an animal knows which stimuli in the environment can alleviate a particular state of deprivation. For example, why does a food-deprived animal approach food specifically, and not water or a sexual partner? This issue of response specificity is relatively easily dealt with in models of motivation that posit multiple, anatomically separable motivational systems, each of which defines a distinct class of stimuli (Coury, 1967; Fisher \& Coury, 1962; Glickman \& Schiff, 1967; Hallonquist \& Mrosovsky, 1986; Roberts, 1969; Wise, 1968). In these models, activation of the hypothalamic feeding substrate exclusively produces food-seeking and feeding behavior, and activation of the drinking substrate elicits water-seeking and drinking behavior. Each stimulus-specific motivational system outputs onto a stimulus-specific behavioral system. Thus, the issue of response specificity is reduced to the activation of a particular, independent motivational system. These models were strongly challenged by the work of Valenstein and Cox (1968), who demonstrated that the specificstimulus-bound behavior elicited by hypothalamic stimulation could be changed to a new behavior with repeated exposures to different goal objects. Thus, it was suggested that the hypothalamus contains a single nonspecific motivational mechanism that is subject to change through experience (see Valenstein, 1969). In terms of the prepotency hypothesis (Cox \& Valenstein, 1969; Valenstein, 1969), response specificity was due to an animal's prior history

Correspondence concerning this article should be sent to K. Nader, Neurobiology Research Group, Department of Anatomy, Faculty of Medicine, University of Toronto, Toronto, ON, Canada M5S 1A8. with a given stimulus; no response specificity was inherent in the neural substrate of motivation.

The discussion of response specificity remains problematic today for two reasons. First, Bindra's proposal of an incentive motivation theory divorced the production of a central motive state from any necessary, specific motor behavior (Bindra, 1976, 1978). If motivation and behavior are two separate processes, it is necessary to explain how motivation may influence behavior. As opposed to its place in earlier models, that of response specificity in incentive motivational theory has to be described without reference to direct activation of a specific motor behavior. Incentive motivational theorists redefine the relationship between the response specificity with respect to certain goal objects and different central motive states in terms of a feed-forward mechanism (Bindra, 1968, 1974, 1976, 1978; Toates, 1986). For example, if a light is paired with the presentation of food, a positive contingency develops between light onset and food presentation (Bindra, 1974). Upon light onset alone, the central representation of light will excite the central representation of food. In turn, these excited central representations induce a feeding central motive state, which then feeds forward to enhance the central representations of the light and food. These enhanced representations are so salient as to demand attention. Thus, different central motive states have the ability to gate the efficiency of incentives to elicit approach behaviors (Bindra, 1968, 1974, 1976, 1978). However, it remains unclear whether feedforward gating of an unconditioned incentive stimulus (such as food in the preceding example) is dependent on a learned association between the central motive state and the perceptual representation of the stimulus, or whether 
the gating of the unconditioned incentive stimulus is an inherent property of motivational systems.

The second reason why response specificity remains problematic is that numerous neurobiological studies suggest that dopamine plays a role in mediating the motivational effects of many natural as well as pharmacological stimuli (see Wise, 1982). If a single neural substrate mediates the unconditioned motivational effects of different classes of stimuli, why does an animal approach food when it is hungry and approach drugs when it is in drug withdrawal? The same problem has arisen in work from our lab demonstrating common substrates for the motivational effects of different classes of stimuli. Place conditioning procedures in conjunction with pharmacological and anatomical manipulations revealed that the brainstem tegmental pedunculopontine (TPP) nucleus and dopamine subserve double dissociable motivational systems that mediate the motivational effects of various classes of stimuli and define a boundary between nondeprived and deprived motivational states. Bilateral lesions of the TPP block the conditioned preferences of food-sated or drugnaive (i.e., nondeprived) animals for environments previously paired with either food or opiates, respectively (Bechara \& van der Kooy, 1989, 1992a; Nader, Harrington, Bechara, \& van der Kooy, 1991; Olmstead \& Franklin, 1991). If animals are food deprived or opiate withdrawn (i.e., deprived), TPP lesions have no effect on the acquisition of conditioned preferences for environments paired with food or opiates over environments paired with hunger or opiate withdrawal, respectively (Bechara, Harrington, Nader, \& van der Kooy, 1992). These results suggest that the TPP nucleus is crucial for the motivational effects of different classes of stimuli only when animals are in a nondeprived motivational state. However, neuroleptic pretreatment prior to conditioning produces the opposite results. Neuroleptics block the motivational effects of either food deprivation or opiate withdrawal (Bechara et al., 1992; Harrington \& van der Kooy, 1992), but have no effect on the acquisition of conditioned preferences for environments paired with either food or opiates in nondeprived animals (Bechara et al., 1992; Nader et al., 1991). These results demonstrate that dopamine mediates the motivational effects of various classes of stimuli only when animals are in deprived, but not in nondeprived, motivational states. Again the question arises, if single neural substrates mediate both the motivational effects of food and drugs, why do animals approach food as opposed to drugs when food deprived?

There are two possible mechanisms that can explain the specificity of goal-directed behaviors, given that the production of a central motive state is not rigidly tied to any particular behavior and that individual motivational systems can mediate the motivational effects of different classes of stimuli. First, motivational systems (such as the TPP- or dopamine-based systems) may be microstructured in such a way that only food can alleviate food deprivation and only drugs can alleviate drug withdrawal (Figure 1A). This implies a high degree of specificity at the level of a motivational system with regard to which stim- uli can relieve particular states of deprivation. Second, the motivational systems may be nonspecific, so that even in the presence of a state of severe food deprivation, the motivational effects of other classes of stimuli may be capable of substituting for the motivational effects of food by relieving the aversive consequences of hunger (Figure 1B). This lack of motivational specificity would necessitate that animals learn which stimulus type will alleviate a particular state of deprivation.

In order to test for specificity at the level of motivational systems, we trained animals by a counterbalanced, unbiased place conditioning procedure (Bechara \& van der Kooy, 1992a; Mucha \& Iverson, 1984; van der Kooy, 1987). It has been demonstrated that these procedures provide sensitive assays of the motivational effects of natural reinforcers as well as psychoactive drugs (see Carr, Fibiger, \& Phillips, 1989). In the animals' training, one

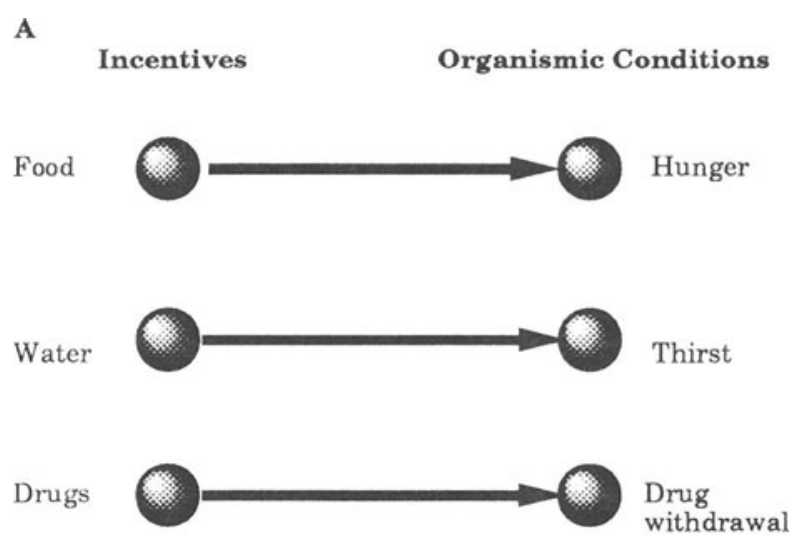

B

Incentives Organismic Conditions

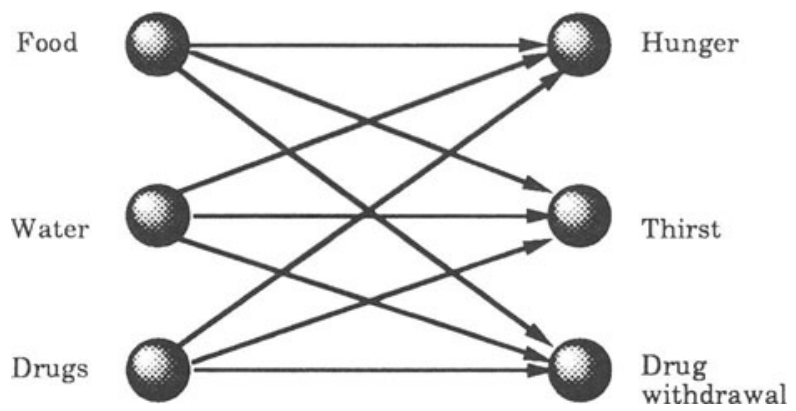

Figure 1. Two possible ways in which motivational systems are structured. (A) There may be an inherent specificity in motivational systems. In this case, only food can alleviate the motivational effects of food deprivation; only water, the motivational effects of thirst; and only drugs, the motivational effects of drug withdrawal. (B) Alternatively, there may be no specificity in motivational systems, so that the motivational effects of any stimulus type can alleviate the motivational effects of a particular type of deprivation. Thus, the motivational effects of a drug like morphine can substitute for food by alleviating the motivational effects of hunger. 
of the two distinctive conditioning environments was paired with $18 \mathrm{~h}$ of food deprivation; the alternate environment was paired with $18 \mathrm{~h}$ of food deprivation and a $20-\mathrm{mg} / \mathrm{kg}$ injection of morphine. If specificity exists at the level of motivational systems, morphine's unconditioned motivational effects in a food-deprived animal should be mediated by the TPP. Although these animals would be food deprived, they also would be drug naive, and, as has been shown previously, the motivational effects of morphine in a drug-naive animal are TPP dependent (Bechara et al., 1992; Bechara \& van der Kooy, $1989,1992 a)$. If there is specificity at the level of motivational systems, then, on the test day, we would predict that animals would display a TPP-dependent preference for the environment paired with morphine and hunger. Alternatively, if motivational systems are structured nonspecifically, the motivational effects of morphine should substitute for the motivational effects of food in a fooddeprived animal. The second hypothesis predicts that, in the same way as food reward in a hungry animal is mediated by dopamine (Horvitz \& Ettenberg, 1988; Spyraki, Fibiger, \& Phillips, 1982a; Wise, Spindler, de Wit, \& Gerber, 1978), morphine's motivational effects in fooddeprived animals should be dependent on dopamine, in spite of the opiate-naive state of the animal.

\section{METHOD}

\section{Subjects}

Adult male Wistar rats weighing 350-450 g (Charles River) were individually housed in suspended gray mesh cages and kept in a temperature- and light-controlled environment (12:12-h light:dark). Throughout the duration of the experiments, all the animals had continuous access to water. Unless otherwise specified, the animals had continuous access to food.

\section{Procedure}

Surgery. Two groups of animals were anesthetized with a $65-$ $\mathrm{mg} / \mathrm{kg} / \mathrm{ml}$ dose of sodium pentobarbital. Using standard stereotaxic techniques, the tip of a 1- $\mu$ l Hamilton syringe was guided into the region of the TPP nucleus. The coordinates, as taken from Paxino and Watson (1982) (with the mouthbar set at $-3.3 \mathrm{~mm}$ below the interaural line), were AP $-7.8 \mathrm{~mm}$ posterior to bregma, $\mathrm{L} \pm$ $1.6 \mathrm{~mm}$ from the midline, and DV $-5.8 \mathrm{~mm}$ below the dura. The animals received bilateral $0.2-\mu$ infusions of either a $4 \%$ ibotenic acid solution (lesion group) or the physiological saline vehicle (sham group) over a 25 -min period. The syringe was left in place for $5 \mathrm{~min}$ following the termination of each infusion. All animals were given at least 2 weeks to recover from the surgery before training commenced.

Histology. Upon completion of the behavioral experiments, all operated rats were anesthetized with an overdose of sodium pentobarbital and perfused transcardially with saline followed by a $10 \%$ formalin solution. The brains were removed and postfixed in $20 \%$ sucrose. Coronal cryostat sections, $32 \mu \mathrm{m}$ thick, were mounted on gelatin coated slides and stained with cresyl violet in order to verify the placement and extent of the lesions. Sections were photographed in bright-field microscopy.

Place conditioning. An unbiased, counterbalanced place conditioning procedure was used for all behavioral experiments. Animals were conditioned in one of two distinct environments that differed in color, smell, and texture (described in detail in Bechara \& van der Kooy, 1992a). Briefly, one box was black, with a Plexi- glas floor that was wiped with a $2 \%$ acetic acid solution before each training session. The other training environment had white walls with a wire-mesh floor that was covered with wood chips. The test box consisted of environments that were exactly the same as the two training environments, but were separated by a narrow gray area on which an animal was placed at the start of each test session.

Prior to training, all rats were handled for a minimum of 5 days. During training, both the order of drug injections and the order of presentation of drug-paired environments were counterbalanced. Upon completion of training, animals received 3 days of continuous access to food and water before testing. At testing, each rat was given free access to the test box and the time spent in each of the two distinct environments was recorded over a 10 -min period.

Morphine place preferences in food-deprived rats after TPP lesions. Two groups of animals, TPP $(n=11)$ and sham $(n=12)$ lesioned, were trained with a standard place conditioning procedure (hereafter referred to as the $B$-procedure, because the animals received equivalent exposures to both conditioning environments) (Bechara and van der Kooy, 1992a; Mucha, van der Kooy, O'Shaughnessy, \& Bucenieks, 1982). Each rat was food deprived for $18 \mathrm{~h}$ and then placed in one of the two conditioning boxes for $45 \mathrm{~min}$. This procedure was repeated four times over an 8-day period. On the 4 alternate days, the same animals were again food deprived for $18 \mathrm{~h}$ and given a $20-\mathrm{mg} / \mathrm{kg}$ i.p. injection of morphine sulphate immediately prior to being placed in the other conditioning environment for $45 \mathrm{~min}$. After a 3-day recovery period, the animals were tested for their preferences for either the environment previously paired with food deprivation or the environment previously paired with food deprivation and morphine. Food-sated and drug-naive animals trained with this procedure and dose of morphine demonstrate TPP-dependent place preferences for the environment paired with morphine (Bechara et al., 1992; Bechara \& van der Kooy, 1989, 1992a).

Effects of neuroleptic pretreatment on morphine's rewarding effects in food-deprived animals. Owing to the findings that morphine can condition a TPP-independent place preference in hungry animals, a modified version of the B-procedure was used to isolate and assess whether or not morphine's rewarding motivational effects in food-deprived animals are dopamine mediated. This procedure (the $M$-procedure) entailed conditioning the animals to only one of the two conditioning environments on four trials equally spaced over 8 days. Although animals were only being conditioned to one environment, the preferences measured by the $\mathrm{M}$ - and Bprocedures are equivalent (Bechara \& van der Kooy, 1992a; Mucha et al., 1982). Prior to conditioning, all the animals were food deprived for $18 \mathrm{~h}$ and received a $20-\mathrm{mg} / \mathrm{kg}$ i.p. morphine injection immediately before being placed in the conditioning environment. On alternate days, the rats were deprived of access to food in their home cage for $18 \mathrm{~h}$, but never exposed to the other conditioning environment. Two and a half hours prior to each conditioning session, the animals were pretreated with either a $0.8-\mathrm{mg} / \mathrm{kg}$ i.p. injection of the neuroleptic alpha-flupentixol $(n=8)$ or its saline vehicle $(n=7)$. At the completion of training, the animals were given a 3-day recovery period with continuous access to food and water. The testing procedure was identical to that previously described for the B-procedure. Previous experiments have shown that rats will acquire a strong place aversion to an environment that has been previously paired with 18 -h food deprivation. Furthermore, this place aversion is dopamine dependent (Bechara et al., 1992; Harrington \& van der Kooy, 1992).

On test day, the animals had a choice between an environment previously paired with morphine's rewarding effects and food deprivation and a novel neutral environment. The animals' preferences, therefore, would be due strictly to morphine's rewarding effects during food deprivation. This contrasted with the B-procedure, in which an animal's preference for a conditioned environment was the result of both morphine's rewarding effects during food deprivation previously paired with one environment (appetitive response) 
and the aversive motivational effects of food deprivation paired to the alternative environment (avoidance response). The M-procedure allowed us to dissociate these two motivational responses and individually test the preferences produced by one of these motivational effects without the possible confounding influence of the other. It is important to note that no motivational effects were attributable to the novelty of the unpaired environment, as revealed in several M-procedure experiments done with the identical apparatus (Bechara et al., 1992; Mucha et al., 1982). Using procedures and apparatus identical to those used in this study, Mucha et al. (1982) showed that rats do not prefer a novel environment over an environment that they have previously been exposed to. Furthermore, on test day no preferences were observed when one of the conditioning environments was paired with a saline injection or the absence of food and the other environment was novel in food-sated rats. Thus, our paradigm was insensitive to confounding effects due to novelty.

\section{RESULTS}

Rats that received bilateral ibotenic acid lesions of the TPP nucleus appeared grossly normal under superficial visual examination and maintained normal body weight in comparison with that of the sham lesioned animals. All animals that were included in the statistical analyses of the behavioral data were histologically verified for the placements and extents of lesions. A representative histological section of a TPP-lesioned rat is shown in Figure 2.

All animals used in these behavioral experiments spent comparable amounts of time in the gray neutral zone that separated the two conditioning environments on the test day. For this reason, and for simplicity of presentation, the scores used in the statistical analyses and presented in the figures do not include times in the gray neutral zone.

\section{Morphine Place Preferences in Food-Deprived Animals After TPP Lesions}

The sham-operated group showed a significant preference $[t(11)=6.8, p<.05]$ for the environment paired with morphine and food deprivation over the environment paired with only food deprivation (Figure 3 ). This demonstrates that the motivational systems of these animals remain sensitive to the rewarding effects of a stimulus that is irrelevant to their physiological state of food deprivation. The TPP-lesioned group demonstrated a similar significant preference $[t(10)=8.4, p<.05]$ for the environment that was paired with morphine and food deprivation. An analysis of variance (ANOVA) of the data from the lesioned and the sham groups revealed that there was no significant interaction of the lesions with the times spent on the morphine- and hunger-versus hunger-paired sides $[F(1,21)=0.42, p>.05]$. Thus, although animals were in a drug-naive state, the motivational effects of morphine in food-deprived animals were not dependent on the TPP nucleus.

\section{Effects of Neuroleptic Pretreatment on Morphine's Rewarding Effects in Food-Deprived Animals}

The saline-pretreated animals showed a significant preference $[t(6)=3.9, p<.05]$ for the environment paired with morphine and hunger over the neutral environment (Figure 4). This finding replicates the earlier results demonstrating that morphine's rewarding motivational effects are expressed in animals that are food deprived. The group that was pretreated with alpha-flupentixol $2.5 \mathrm{~h}$ prior to conditioning, however, showed no significant conditioned

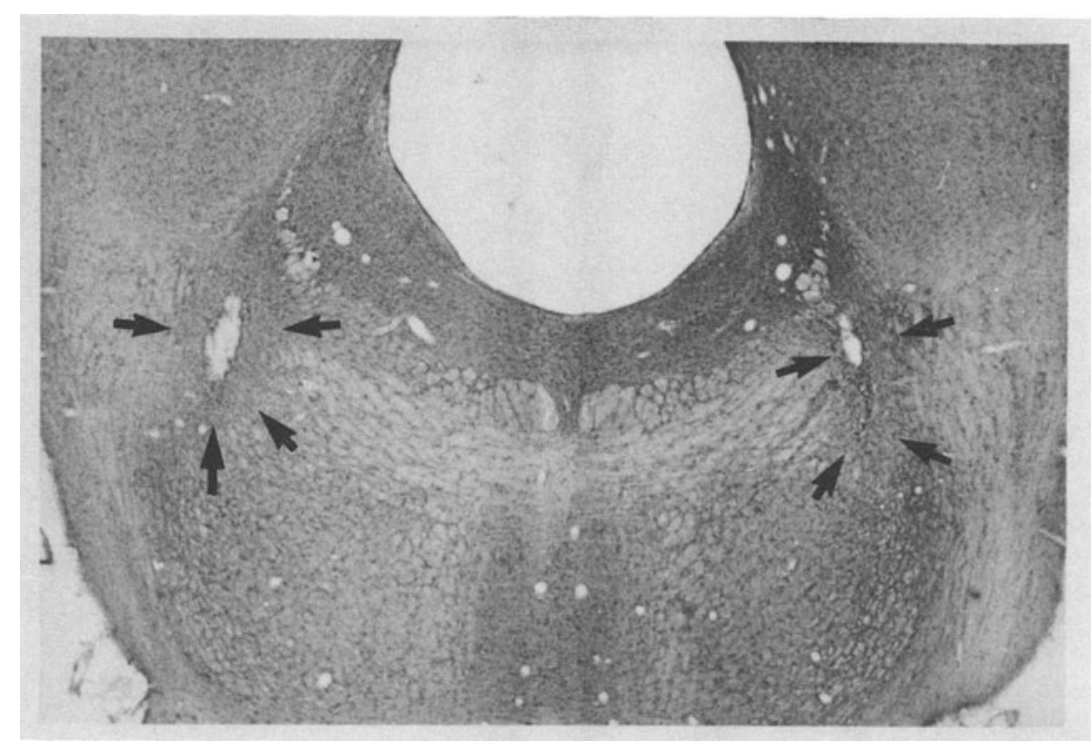

Figure 2. A photomicrograph of a Nissl-stained coronal section through the pons showing bilateral ibotenic acid lesions (arrows) of the tegmental pedunculopontine nucleus. The lesions show gross bilateral symmetry and were easily identified by the loss of neuronal perikarya and the presence of gliosis. 


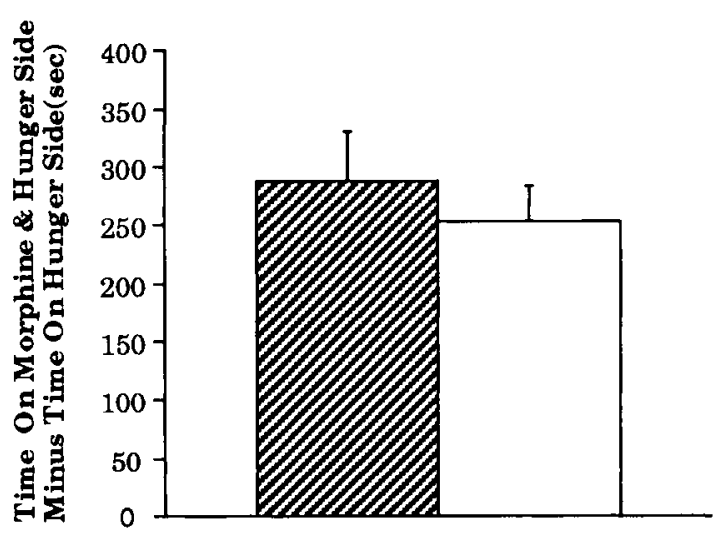

Sham Lesion TPP Lesion

Figure 3. Effects of bilateral ibotenic acid or sham tegmental pedunculopontine (TPP) lesions on place conditioning, as assessed by the B-procedure, in rats that were exposed to one environment after $18 \mathrm{~h}$ of food deprivation and the alternate environment after $18 \mathrm{~h}$ of food deprivation and a $20-\mathrm{mg} / \mathrm{kg}$ i.p. injection of morphine. Bars represent means $\pm S E M$.

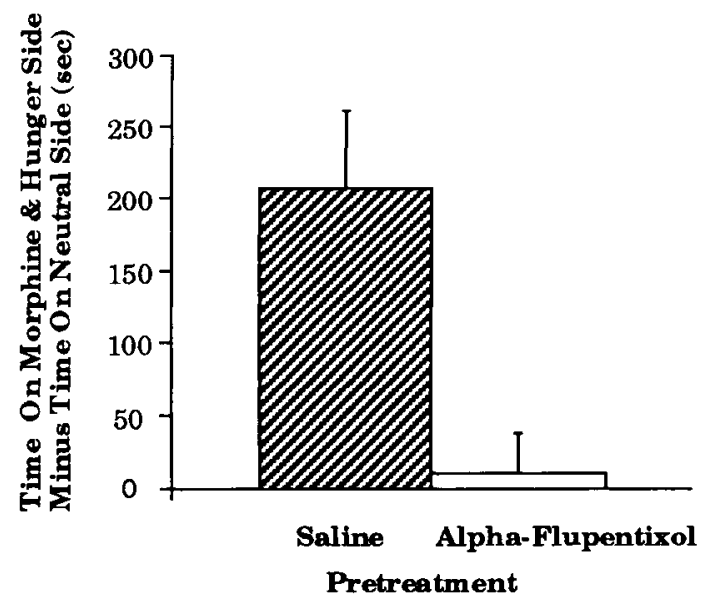

Figure 4. Effects of alpha-flupentixol (0.8-mg/kg i.p.) or saline vehicle pretreatment $2.5 \mathrm{~h}$ prior to conditioning on the conditioned place preferences produced by a $20-\mathrm{mg} / \mathrm{kg}$ i.p. injection of morphine in 18-h food-deprived rats. All animals were conditioned according to the M-procedure, such that on the test day, rats had a choice between (1) a morphine and hunger paired environment and (2) a novel, neutral environment. Bars represent means \pm SEM.

preference $[t(7)=0.4, p>.05]$ for either environment. An ANOVA of the side preferences of neuroleptic- and saline-pretreated rats revealed a significant interaction $[F(1,13)=11.98, p<.05]$ of neuroleptic pretreatment with the times spent in the morphine- and hunger-paired environment versus the neutral environment. Thus, morphine's rewarding effects in food-deprived animals were mediated by the dopaminergic, deprivation-dependent, motivational system.

\section{DISCUSSION}

The motivational effect of morphine can substitute for food in food-deprived animals. Rats showed significant preferences for an environment paired with morphine and food deprivation over an environment paired with food deprivation alone. These preferences were blocked by neuroleptic pretreatment and not by TPP lesions. The finding that morphine's motivational effects were mediated by the dopaminergic, deprivation-dependent, motivational system, which is specifically activated by food deprivation (Bechara et al., 1992; Harrington \& van der Kooy, 1992), suggests that morphine was substituting for food and not simply producing its normal (drug-naive) unconditioned effects via a separate motivational system. Had morphine been producing its normal (drug-naive) unconditioned effects, we would have expected the observed preferences to be blocked by TPP lesions and not neuroleptic pretreatment. Although the rats were food deprived, they were also drug naive, and morphine's unconditioned rewarding effects in drug-naive and food-sated animals have previously been shown to be dependent on the TPP nucleus and not dopamine (Bechara et al., 1992; Bechara \& van der Kooy, 1989, 1992a; Mackey \& van der Kooy, 1985; Olmstead \& Franklin, 1991). Furthermore, the finding that TPP lesions had no effect on the acquisition of preferences for an environment paired with morphine and food deprivation extends previous findings demonstrating that the TPP (opiate-nondeprived) motivational system is specifically overshadowed in opiate-deprived animals (Bechara \& van der Kooy, 1992b). Because the blockade by neuroleptic pretreatment of the motivational effects of opiate deprivation continues to mask TPPmediated, nondeprived motivational effects when animals are in opiate withdrawal, it has been suggested that the mechanism by which overshadowing occurs is nonmotivational (Bechara \& van der Kooy, 1992b). The results presented here suggest that this overshadowing of the nondeprived motivational system when animals are in a deprived state is not restricted to the unconditioned effects of only those stimuli from which the animal is deprived, but is generalizable across stimulus classes. Traditionally, overshadowing has referred to a situation in which two conditioned stimuli (CSs) are concurrently paired with an unconditioned stimulus (US). Only one of the two CSs will come to elicit a conditioned response. Thus, the CS that acquired a predictive relationship with the US is said to have overshadowed the other CS (Mackintosh, 1983). In the present context, we use the term to refer to the ability of one motivational system to overshadow the effects of a second motivational system (Bechara \& van der Kooy, 1992b).

From these results, we would predict that food can substitute for the motivational effects of morphine in animals that are in opiate withdrawal. Because of procedural difficulties, however, it is impossible to perform the mirror experiment with food substituting for morphine in rats experiencing opiate withdrawal. This is because animals 
treated with the dose of alpha-flupentixol that was used in these studies are impaired by motor deficits. Thus, rats treated with neuroleptics will not eat in order to sustain a place preference in the first place. On test day, therefore, the rats would have a choice between an environmentpaired withdrawal only and a neutral environment, which we know produces a dopamine-dependent place aversion (Bechara et al., 1992).

An alternate interpretation of these results could suggest that morphine is working through a separate dopaminergic motivational system (independent of the fooddeprivation-activated dopaminergic motivational system) to produce its rewarding effects. Thus, rats that were receiving an injection of morphine would have experienced an aversion due to food deprivation that was equivalent to the one they experienced when injected with saline. This independent dopamine systems interpretation seems unlikely, however, given the demonstrations that peripheral injections of morphine decrease the amount of food consumed in food-deprived (Kunihara, Kanbayashi, \& Ohshima, 1983; Sanger \& McCarthy, 1980) or weightrestricted (Levine, Morley, \& Raij, 1986) rats. In addition, Marks-Kaufman and Kanarek (1980) demonstrated that morphine injections decreased, in a dose-dependent manner, the consumption of calories by food-deprived rats. If the motivational effects of morphine and food deprivation were acting through separate dopaminergic substrates, one would predict no effect of morphine pretreatment on the amount of food consumed. The decrease in consumption seen in these studies cannot be explained in terms of morphine's unconditioned cataleptic effects, because morphine injections of the same doses in naive rats produced heightened food consumption (Kunihara et al., 1983; Lowy, Starkey, \& Yim, 1981; Sanger \& McCarthy, 1980,1981 ).

It is well known that stimulants such as amphetamine produce anorexia (see Hoebel, 1977). Numerous studies have demonstrated that amphetamine partially acts to produce its anorexic effects through a dopaminergic substrate (Baez, 1974; Blundell \& Lathman, 1979; Carruba et al., 1986; Fibiger, Zis, \& McGeer, 1973; Heffner, Zigmond, \& Stricker, 1977; Hollister, Ervin, Cooper, \& Breese, 1975; Leibowitz, 1975). The motivational effects of amphetamine are also dopamine dependent (Hiroi \& White, 1990, 1991; Mackey \& van der Kooy, 1985; Spyraki, Fibiger, \& Phillips, 1982b; Yokel \& Wise, 1976). Our observations that morphine can substitute for food via a dopaminergic (deprivation-dependent) mechanism suggests that perhaps some of the anorexic properties of high doses of amphetamine are due to its ability to substitute for food in food-deprived rats via the dopaminergic (deprivation-dependent) motivational system (Blundell, Latham, \& Leshem, 1976). Similarly, the ability of one class of stimuli to substitute for another also might explain the increased self-administration of a variety of psychoactive agents in response to food deprivation (see Kanarek \& Marks-Kaufman, 1988).
Given that there is no inherent specificity in motivational systems and that one type of stimulus can substitute for another, we must still explain the fact that hungry animals approach food and not drugs when given the choice. This is especially problematic if both food and drug stimuli have the same short-term effect of alleviating the motivational effects of hunger. A learning mechanism could underlie the observed response specificity without any necessary reference to specificity at the level of motivational systems, or to specificity of the exact behavior elicited. During the ontogeny of an animal, the perceptual (discriminative) effects of food deprivation may come to be strongly associated with food-seeking behaviors. Given that Martin, Bechara, and van der Kooy (1991) have demonstrated that TPP lesions in naive animals block the acquisition of a place preference for an environment paired with morphine but have no effect on the same animal's ability to use morphine as a discriminative cue, it has been suggested that the processes mediating the perceptual and motivational effects of drugs are dissociable. Supporting this dissociation is work by Berridge, Venier, and Robinson (1989) demonstrating that intranigral 6-OHDA lesions that rendered rats aphagic had no effect on an animal's hedonic, perceptual reactions to different flavors. Thus, food consumption should alleviate concurrently the separate perceptual and motivational effects of food deprivation. Through this association in time between the attenuation of the perceptual cues of hunger and food consumption, the perceptual effects of food deprivation may come to act as powerful discriminative cues for appetitive responses to food. Interestingly, in contrast to earlier failed attempts to condition an instrumental response to an internal cue derived from states of deprivation (Bolles, 1975; Webb, 1955), numerous studies have recently demonstrated that the intensity of food deprivation can indeed produce discriminative cues that can control instrumental responses (Capaldi \& Davidson, 1979; Capaldi, Viveiros, \& Davidson, 1981; Corwin, Woolverton, \& Schuster, 1990; Schechter, 1990), as well as enter into Pavlovian conditional discriminations (Davidson, 1987; Davidson, Flynn, \& Jarrard, 1992). Perhaps environmental cues that come to condition feeding behavior (Weingarten, 1985) do so by eliciting the discriminative effects of food deprivation.

Given that the discriminative and motivational effects of stimuli are not isomorphic but dissociable (Berridge et al., 1989; Martin et al., 1991), then drugs may alleviate the motivational, but not the discriminative, effects of hunger. Amphetamine has strong anorexic effects, but minimal, if any, ability to substitute for the perceptual effects of food in a food-deprived animal (Corwin et al., 1990; Schechter, 1990). Therefore, the alleviation of the discriminative effects induced by food deprivation would be specifically correlated only with food ingestion. Although various stimuli in the environment can substitute for the motivational effects of other classes of stimuli in deprived animals, the discriminative effects of the depri- 
vation state should maintain approach behavior (given that several stimulus types are available) only toward the stimulus type from which the animal is deprived.

This association between the discriminative effects of food deprivation and the appetitive response to food is probably learned within the first 2 weeks postparturition, a time when suckling is one of the dominant appetitive behaviors (Alberts \& Gubernick, 1984; E. M. Blass, Hall, \& Teicher, 1979; Henning, 1981). During this period, ingestion of mother's milk will be highly correlated with the alleviation of the perceptual (as well as the motivational) effects of hunger. Even in the first few days of postnatal life, rat pups are capable of appetitive learning (J. T. Blass \& E. M. Blass, 1977; Johanson \& Hall, 1979; Kenny \& Blass, 1977; Moran, Lew, \& Blass, 1981; Phifer, Denzinger, \& Hall, 1992). Associations that have been learned during the suckling period can be extended and utilized during the postweaning period of independent ingestion (Alberts \& Gubernick, 1984). This period of development may function as a Hebbian intermediate stage, when pups learn the close relationship between the alleviation of the perceptual effects of hunger and ingestion (Hebb, 1949). Bindra (1978) postulated that a discriminative stimulus (pexgo) is not necessarily associated with any one particular response; rather, "the (unspecified) response that is produced is a fresh construction dependent on the motivational state and the pexgo generated by the prevailing stimulus situation" (p. 42). Thus, the relationship between the perceptual effects (pexgo) of hunger and food ingestion during the suckling stage of a neonate should generalize across feeding stages to future independent feeding behavior.

This proposed model of directed behavior differs in two ways from the traditional incentive-motivational view positing that a central motive state gates the efficiency of incentive stimuli (Bindra, 1976, 1978). First, our model uniquely suggests a central role for the association that is formed between the perceptual properties of deprivation and stimuli that concurrently alleviate the separate perceptual and the motivational effects of that deprivation. Second, the ability of a particular central motive state to feed forward and enhance the central representation of a particular incentive stimulus is difficult to reconcile with our findings that no specificity exists at the level of motivational systems. In the incentive motivational view, it is hard to imagine how a nonspecific motivational system can specifically enhance the central representation of the stimulus from which the animal is deprived. The model presented here dissociates the motivational and perceptual effects of deprivation, allowing rewarding stimuli in the environment to alleviate both effects and, in doing so, to closely associate the distinct perceptual effects of one type of deprivation with the rewarding stimuli in the environment that alleviate these perceptual effects. Implicit in our model is that a discussion of response specificity is restricted to the behavior of deprived animals. Rats in a nondeprived, TPP-mediated state that lacks the perceptual effects of deprivation should engage in behavior that is explorative, rather than specific, in nature.
In conclusion, we suggest that no stimulus-response specificity exists in the neural substrate of motivation. Rather, the motivational effects of various stimulus classes can each alleviate the motivational effects of any particular state of deprivation via a dopaminergic (deprivationdependent) motivational system. A corollary of these observations is that the ability of a state of deprivation to overshadow the TPP (nondeprived) motivational system is generalizable across stimulus boundaries. We suggest that during the normal ontogeny of an animal, the discriminative (nonmotivational) effects of deprivation come to be associated with the appropriate stimulus type that can alleviate that particular state of deprivation. This is the result of food ingestion concurrently overcoming the separate motivational and discriminative effects of hunger. Thus, the perceptual effects of deprivation will acquire conditioned control over which stimulus type to approach, and the motivational effects of deprivation will energize the animal.

\section{REFERENCES}

Alberts, J. R., \& Gubernick, D. J. (1984). Early learning as ontogenetic adaptation for ingestion by rats. Learning \& Motivation, 15, 334-359.

BAEZ, L. A. (1974). Role of catecholamines in the anorectic effects of amphetamines in rats. Psychopharmacology, 35, 91-98.

Baile, C. A., Mclaughlin, C. L., \& Della-Fera, M. Z. (1986). Control of food intake. Physiological Review, 66, 172-234.

Bechara, A., Harrington, F., Nader, K., \& van der Kooy, D. (1992). The neurobiology of motivation: Double dissociation of two motivational mechanisms mediating opiate reward in naive versus drug dependent animals. Behavioral Neuroscience, 106, 798-807.

Bechara, A., \& VAN DER KoOY, D. (1989). The tegmental pedunculopontine nucleus: A brain-stem output of the limbic system critical for the conditioned place preferences produced by morphine and amphetamine. Journal of Neuroscience, 9, 3400-3409.

BECHARA, A., \& VAN DER KOOY, D. (1992a). A single brain substrate mediates the motivational effects of both opiates and food in nondeprived, but not in deprived animals. Behavioral Neuroscience, 106, 351-363.

Bechara, A., \& VAN der KOOY, D. (1992b). Chronic exposure to morphine does not alter the neural tissue subserving its acute rewarding properties: Apparent tolerance is overshadowing. Behavioral Neuroscience, 106, 364-374.

Berridge, K. C., Venier, I. L., \& Robinson, T. E. (1989). Taste reactivity analysis of 6-hydroxydopamine-induced aphagia: Implications for arousal and anhedonia hypotheses of dopamine function. Behavioral Neuroscience, 103, 36-45.

BindRA, D. (1968). Neuropsychological interpretation of the effects of drive and incentive-motivation on general activity and instrumental behavior. Psychological Review, 75, 1-22.

Bindra, D. (1974). Motivational view of learning, performance, and behavior modification. Psychological Review, 81, 199-213.

BINDRA, D. (1976). A theory of intelligent behavior. New York: Wiley. BINDRA, D. (1978). How adaptive behavior is produced: A perceptualmotivational alternative to response-reinforcement. Behavioral \& Brain Sciences, 1, 41-91.

Blass, E. M., Hall, W. G., \& Teicher, M. H. (1979). The ontogeny of suckling and ingestive behaviors. Progress in Psychobiology \& Physiological Psychology, 8, 243-299.

Blass, J. T., \& Blass, E. M. (1977). Suckling as incentive to instrumental learning in preweanling rats. Science, 196, 898-899.

Blundell, J. E., \& LATHam, C. J. (1979). Characterization of adjustments to the structure of feeding behavior following pharmacological treatment: Effects of amphetamine and fenfluramine and the an- 
tagonism produced by pimozide and methergoline. Pharmacology, Biochemistry \& Behavior, 12, 717-722.

Blundell, J. E., Latham, C. J., \& Leshem, M. B. (1976). Differences between the anorexic actions of amphetamine and fenfluraminepossible effects on hunger and satiety. Journal of Pharmacy \& Pharmacology, 28, 471-477.

Bolles, R. C. (1975). Theory of motivation. New York: Harper \& Row.

Capaldi, E. D., \& Davidson, T. L. (1979). Control of instrumental behavior by deprivation stimuli. Journal of Experimental Psychology: Animal Behavior Processes, 5, 355-367.

Capaldi, E. D., Viveiros, D. M., \& Davidson, T. L. (1981). Deprivation stimulus intensity and incentive factors in the control of instrumental responding. Journal of Experimental Psychology: Animal Behavior Processes, 7, 140-149.

Carr, G. D., Fibiger, H. C., \& Phillips, A. G. (1989). Conditioned place preference as a measure of drug reward. In J. M. Liebman \& S. J. Cooper (Eds.), The pharmacological basis of reward (pp. 264319). New York: Oxford University Press.

Carruba, M. O., Coen, E., Pizzi, M., Memo, M., Missale, C., Spano, P. F., \& ManTEgazza, P. (1986). Mechanisms of action of anorectic drugs: An overview. In M. O. Carruba \& J. E. Blundell (Eds.), Pharmacology of eating disorders (pp. 1-29). New York: Raven Press.

Corwin, R. L., Woolverton, W. L., \& Schuster, C. R. (1990). Effects of cholecystokinin, $d$-amphetamine and fenfluramine in rats trained to discriminate 3 from $22 \mathrm{hr}$ of food deprivation. Joumal of Pharmacology \& Experimental Therapeutics, 253, 720-728.

Coury, J. N. (1967). Neural correlates of food and water intake in the rat. Science, 156, 1763-1765.

Cox, V. C., \& VALENSTEIN, E. S. (1969). Distribution of hypothalamic sites yielding stimulus-bound behavior. Brain, Behavior \& Evolution, 2, 359-376.

DAVIDSON, T. L. (1987). Learning about deprivation intensity stimuli. Behavioral Neuroscience, 101, 198-208.

Davidson, T. L., Flynn, F. W., \& Jarrard, L. E. (1992). Potency of food deprivation intensity cues as discriminative stimuli. Journal of Experimental Psychology: Animal Behavior Processes, 18, 174-181.

Fibiger, H. C., Zis, A. P., \& McGeer, E. G. (1973). Feeding and drinking deficits after 6-hydroxydopamine administration in the rat: Similarities to the lateral hypothalamic syndrome. Brain Research, 55, $135-148$.

Fisher, A. E., \& Coury, J. N. (1962). Cholinergic tracing of a central neural circuit underlying the thirst drive. Science, 138, 691.

GLICKMAN, S. E., \& SCHIFF, B. B. (1967). A biological theory of reinforcement. Psychological Review, 74, 81-109.

Hallonquist, J. D., \& Mrosovsky, N. (1986). Electrically induced behavior and neural specificity in ground squirrels and dormice. Physiology \& Behavior, 38, 387-397.

Harrington, F., \& VAN DER Kooy, D. (1992). Deprivation state determines the motivational effects of neuroleptics in rats. Psychobiology, 20, 294-299.

HeBB, D. O. (1949). Organization of behavior. New York: Wiley.

HeffNer, T. G., Zigmond, M. J., \& STricker, E. M. (1977). Effects of dopaminergic agonists and antagonists on feeding in intact and 6hydroxydopamine-treated rats. Joumal of Pharmacology \& Experimental Therapeutics, 201, 386-399.

Henning, S. J. (1981). Postnatal development: Coordination of feeding, digestion, and metabolism. American Journal of Physiology, 241, G199-G214.

HIROI, N., \& WhITE, N. M. (1990). The reserpine-sensitive dopamine pool mediates $(+)$-amphetamine-conditioned reward in the place preference paradigm. Brain Research, 510, 33-42.

HIROI, N., \& WhITE, N. M. (1991). The amphetamine conditioned place preference: Differential involvement of dopamine receptor subtypes and two dopaminergic terminal areas. Brain Research, 552, 141-152.

HoEbeL, B. G. (1977). The psychopharmacology of feeding. In L. L. Iversen, S. D. Iversen, \& S. H. Snyder (Eds.), Handbook of psychopharmacology (pp. 55-129). New York and London: Plenum.

Hollister, A. S., Ervin, G. N., Cooper, B. R., \& Breese, G. R. (1975). The roles of monoamine neural systems in the anorexia induced by $(+)$-amphetamine and related compounds. Neuropharmacology, 14, 715-723.
Horvitz, J. C., \& Ettenberg, A. (1988). Haloperidol blocks the response-reinstating effects of food reward: A methodology for separating neuroleptic effects on reinforcement and motor processes. Pharmacology, Biochemistry \& Behavior, 31, 341-350.

Johanson, I. B., \& HALL, W. G. (1979). Appetitive learning in 1-dayold rat pups. Science, 205, 419-421.

Kanarek, R. B., \& Marks-Kaufman, R. (1988). Animal models of appetitive behavior: Interaction of nutritional factors and drug seeking behavior. In M. Winick (Ed.), Control of appetite (pp. 1-25). New York: Wiley.

Kenny, J. T., \& Blass, E. M. (1977). Suckling as incentive to instrumental learning in preweanling rats. Science, 196, 898-890.

Kunihara, M., Kanbayashi, M., \& Ohshima, T. (1983). Opposite effects of morphine on feeding and drinking in rats relative to administration time. Japanese Journal of Pharmacology, 33, 829-835.

LEIBOWITZ, S. F. (1975). Catecholaminergic mechanisms of the lateral hypothalamus: Their role in the mediation of amphetamine anorexia. Brain Research, 98, 529-545.

Levine, A. S., Morley, J. E., \& RaiJ, L. (1986). Opiates and the anorexia of uremia. Physiology \& Behavior, 37, 835-838.

Lowy, M. T., StarkeY, T. C., \& YIM, G. K. W. (1981). Stereoselective effects of opiate agonists on ingestive behavior in rats. Pharmacology, Biochemistry \& Behavior, 15, 591-596.

MACKEY, W. B., \& VAN DER KOOY, D. (1985). Neuroleptics block the positive reinforcing effects of amphetamine but not morphine as measured by place conditioning. Pharmacology, Biochemistry \& Behavior, 22, 101-105.

MaCkintosh, N. J. (1983). Laws of association. In N. J. Mackintosh (Ed.), Conditioning and associative learning (pp. 42). New York: Oxford University Press.

Marks-Kaufman, R., \& Kanarek, R. B. (1980). Morphine selectively influences macronutrient intake in the rat. Pharmacology, Biochemistry \& Behavior, 12, 427-430.

Martin, G. M., Bechara, A., \& Van der Kooy, D. (1991). The perception of emotion: Parallel neural processing of the affective and discriminative properties of opiates. Psychobiology, 19, 147-152.

Moran, T. H., LEW, M. F., \& Blass, E. M. (1981). Intracranial selfstimulation in 3-day-old rat pups. Science, 214, 1366-1368.

MuCHA, R. F., \& IVERSEN, S. D. (1984). Reinforcing properties of morphine and naloxone revealed by conditioned place preference: A procedural examination. Psychopharmacology, 82, 241-247.

Mucha, R. F., Van der Kooy, D., O'Shaughnessy, M., \& BuceNIEKS, P. (1982). Drug reinforcement studies by the use of place conditioning in rat. Brain Research, 243, 91-105.

Nader, K., Harrington, F., Bechara, A., \& van der Kooy, D. (1991). Neuroleptics block the rewarding effects of high, but not low, doses of heroin in drug naive animals. Sociery for Neuroscience $A b$ stracts, 17, 1048.

Olmstead, M. C., \& Franklin, K. B. J. (1991). Effects of pedunculopontine tegmental nucleus lesions on morphine induced conditioned place preference and analgesia in the formalin test. Society of Neuroscience Abstracts, 17, 1433.

Paxino, G., \& Watson, C. (I982). The rat brain in stereotaxic coordinates. San Diego, CA: Academic Press.

Phifer, C. B., Denzinger, A., \& Hall, W. G. (1992). The early presence of food-oriented appetitive behavior in developing rats. Developmental Psychobiology, 24, 453-461.

ROBERTs, W. W. (1969). Are hypothalamic motivational mechanisms functionally and anatomically specific? Brain, Behavior \& Evolution, 2, 317-342.

SANGer, D. J., \& McCARThY, P. S. (1980). Differential effects of morphine on food and water intake in food deprived and freely-feeding rats. Psychopharmacology, 72, 103-106.

SANGER, D. J., \& MCCARTHY, P. S. (1981). Increased food and water intake produced in rats by opiate receptor agonists. Psychopharmacology, 74, 217-220.

SCHECHTER, M. D. (1990). Comparison of anorectic drugs in rats trained to discriminate between satiation and deprivation. Life Sciences, 47, 17-24.

Spyraki, C., Fibiger, H. C., \& Phillips, A. G. (1982a). Attenuation by haloperidol of place preference conditioning using food reinforcement. Psychopharmacology, 77, 379-382. 
Spyraki, C., Fibiger, H. C., \& Phillips, A. G. (1982b). Dopaminergic substrates of amphetamine-induced place preference conditioning. Brain Research, 253, 185-193.

Toates, F. M. (1986). Motivational systems. Cambridge: Cambridge University Press.

VALENSTEIN, E. S. (1969). Behavior elicited by hypothalamic stimulation. Brain, Behavior \& Evolution, 2, 295-316.

VAlENSTEIN, E. S., \& Cox, V. C. (1968). Modification of motivated behavior elicited by electrical stimulation of the hypothalamus. Science, 159, 1119-1121.

VAN DER KoOY, D. (1987). Place conditioning: A simple and effective method for assessing the motivational properties of drugs. In M. A. Bozarth (Ed.), Methods of assessing the reinforcing properties of abused drugs (pp. 229-241). New York: Springer-Verlag.

WebB, W. B. (1955). Drive stimuli as cues. Psychological Reports, $1,287-298$.
Weingarten, H. P. (1985). Stimulus control of eating: Implications for a two-factor theory of hunger. Appetite, 6, 387-401.

WiSE, R. A. (1968). Hypothalamic motivational systems: Fixed or plastic neural circuits? Science, 162, 377-379.

WISE, R. A. (1982). Neuroleptics and operant behavior: The anhedonia hypothesis. Behavioral \& Brain Sciences, 5, 39-87.

Wise, R. A., Spindler, J., De Wit, H., \& Gerber, J. G. (1978). Neuroleptic induced "anhedonia" in rats: Pimozide blocks reward quality of food. Science, 201, 262-264.

YoKel, R. A., \& WISE, R. A. (1976). Attenuation of intravenous amphetamine reinforcement by central dopamine blockade in rats. $P$ sychopharmacology, 48, 311-318.

(Manuscript received August 5, 1993; revision accepted for publication October 8, 1993.) 